\section{Rhinitis bei Asthma stärker berücksichtigen}

Fiver ast $80 \%$ der Patienten mit Asthma bronchiale haben auch eine allergische Rhinitis, berichtete Prof. Dr. Winfried Randerath, Solingen. Zwischen den beiden Erkrankungen bestehen gemeinsame Pathomechanismen, die Symptomstärke ist positiv korreliert. Im aktualisierten Positionspapier der Initiative „Allergic Rhinitis and its Impact on Asthma“ (ARIA) wird deshalb gefordert, diesen Zusammenhang bei Diagnostik und Therapie zu beachten.

Prof. Dr. Carl-Peter Criée, Lenglern, wies darauf hin, dass bei der The-

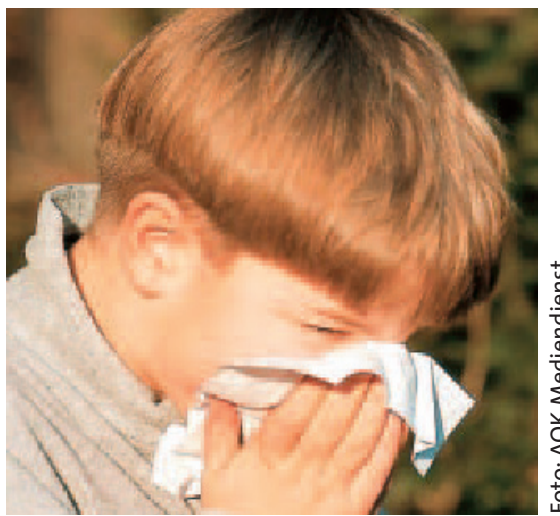

Viele Asthmatiker haben auch Heuschnupfen. rapie des Asthma bronchiale stärker das Konzept der Asthmakontrolle als des Schweregrads im Vordergrund steht. Je nach Symptomkontrolle sollte die bestehende Therapie intensiviert oder beibehalten bzw. die niedrigste mögliche Behandlungsstufe ermittelt werden. Als Dauermedikamente stehen inhalative Glukokortikoide, langwirksame Betamimetika oder der Leukotrienantagonist Montelukast (Singulair ${ }^{\circledR}$ ) zur Verfügung. Montelukast kann bei Kindern zwischen zwei und 14 Jahren bereits als erster Controller eingesetzt werden. Dies empfiehlt auch die European Pediatric Asthma Consensus Group. Beim Infektasthma, das bei Kindern im (Vor)schulalter häufig auftritt, ist Montelukast auch als Kurzzeittherapie wirksam, so Prof. Dr. Jürgen Seidenberg, Oldenburg. Der Leukotrienantagonist ist dabei gegenüber inhalativen Glukokortikoiden im Vorteil, da er schneller eine Bronchodilatation bewirkt. af

Symposium „Asthma - bekannte Pro-

bleme, neue Lösungen?" 49. Kongress der deutschen Gesellschaft für Pneumologie, Lübeck, 9.-12. April 2008.

Veranstalter: MSD, Haar

\section{Hilfsmittelnummer für Spacer}

Der ABLE SPACER ${ }^{\circledR}$ kann jetzt unter der Positions-Nr. 14.24.03.1001 als Hilfsmittel abgerechnet werden. Das machten die Spitzenverbände der Krankenkassen im Nachtrag zum Hilfsmittelverzeichnis vom 1. August 2007, veröffentlicht im Bundesanzeiger Nr. 170 vom 11.9.2007, bekannt. Der ABLE SPACER ${ }^{\circledR}$ belastet somit nicht das Arzneimittelbudget des Arztes, da er als Hilfsmittel nicht budgetiert ist.

Nach Informationen von Dr. Beckmann Pharma, Seefeld

\section{Unternehmen neu zertifiziert}

Das biopharmazeutische Unternehmen Stallergenes $\mathrm{GmbH}$ erhielt am 26. Februar 2008 offiziell das ISO-Zertifikat der Qualitätsnorm ISO 9001:2000. Damit macht das Unternehmen seinen hohen Anspruch an Qualität deutlich. Qualitätsmanagement ist nach eigenem Bekunden ein wichtiger Bestandteil der Firmenpolitik und gilt auf allen Ebenen, von der Herstellung der Allergieextrakte bis zur Dienstleistung. Bei dem DIN-ISO Zertifizierungsverfahren wurden alle relevanten Unternehmensabläufe überprüft.

Nach Informationen von Stallergenes, Kamp-Linfort

\title{
Preisvergabe für Komplett-Therapie
}

E rstmals wurde in diesem Jahr der Preis für „Herausragende Therapiekonzepte in der Pneumologie" vergeben. Das Präparat POLLINEX ${ }^{\circledR}$ Quattro erhielt den vom Kölner Biermann Verlag ausgelobten Preis.

Ermittelt wurde das Preisträgerpräparat durch eine unabhängige Jury, die Präparate, die in der spezifischen Kurzzeit-Immuntherapie zum Einsatz kommen, bewertete. Kriterien waren unter anderem Anwender- und Patientenfreundlichkeit, Therapiekosten und klinische Evidenz. Jurymitglied Prof. KarlChristian Bergmann, Berlin, begründete die Preisvergabe an POLLINEX $^{\circledR}$ Quattro mit dem Fortschritt, den dieses Produkt zur subkutanen spezifischen
Immuntherapie mit nur vier Injektionen in drei Wochen pro Jahr für viele Patienten gebracht hat. Ermöglicht wird die Verkürzung der Behandlungszeit durch den Einsatz des Adjuvans Monophosphoryl-Lipid A $\left(\mathrm{MPL}^{\circledR}\right)$. Das detoxifizierte Lipid A aus der Zellwand von Salmonella minnesota bindet an Toll-like-Rezeptoren(TLR-4), die wie beim Kontakt eines echten Pathogens die körpereigene Abwehr durch Stimulation von Zellfunktionen unterstützen. So fördert das Adjuvans die Immunmodulation im Sinne einer TH1-gerichteten Immunantwort.

Mit der Entwicklung einer Komplett-Therapie mit nur vier Injektionen wurde ein wichtiger Beitrag zur Verbes- serung der Compliance geleistet, was wiederum zu einer besseren und kostengünstigeren Versorgung allergischer $\mathrm{Pa}$ tienten führt, meint Bergmann. Andrea Fischer, Bundesgesundheitsministerin a.D., hob in ihrer Festrede die Bedeutung fortschrittlicher Therapiekonzepte für die Verbesserung der medizinischen Versorgung der Bevölkerung hervor.

Stellvertretend für Bencard Allergie nahm Peter Keysers, Geschäftsführer Bencard Allergie, den Preis im Rahmen des diesjährigen Pneumologenkongresses entgegen. $b k$

Verleihung des Biermann-Preises „Herausragende Therapiekonzepte in der Pneumologie". 49. Kongresses der Deutschen Gesellschaft für Pneumologie und Beatmungsmedizin, 9. bis 12. April 2008. Veranstalter: Biermann-Verlag, Köln 